doi:10.17659/01.2017.0011

Journal of Case Reports 2017;7(1):39-42

\title{
Primary, Spontaneous, Complete Colporrhexis during Precipitate Vaginal Birth after Cesarean Section (VBAC)
}

\author{
Asmaa Abdulsalam, Lakshmi Bayi Sushama, Zeenat Rizvi, Mythili Tallavajhula \\ Department of Obstetrics and Gynecology, Al-Ahli Hospital, P.O.Box 6401, Doha, Qatar.
}

\section{Corresponding Author:}

Dr. Asmaa Abdulsalam

Email: abdulslamasma@gmail.com

This is an Open Access article distributed under the terms of the Creative Commons Attribution License (creativecommons.org/ licenses/by/3.0).

Received Accepted Published

October 26, 2016

December 28, 2016

January 30, 2017

\begin{abstract}
Background: Obstetric hemorrhage emerges as the most common cause of severe maternal morbidity and mortality around the globe. Colporrhexis or rupture of the vaginal vault is a rare, but dangerous complication of labor and is a rare cause of postpartum hemorrhage. Case Report: We hereby report 32 years old second gravida undergoing vaginal birth after cesarean section, who in second stage of labor sustained a spontaneous posterior vaginal wall tear extending in to the posterior fornix opening into the pouch of Douglas, which was managed successfully by both vaginal and abdominal approach. Conclusion: This case-study also highlights the importance of quality of care as one of the determinants of phase III delay affecting the prognosis and outcomes while caring for near misses in obstetrics.
\end{abstract}

Keywords: Labor, Postpartum Hemorrhage, Pregnancy, Vaginal Birth after Cesarean.

\section{Introduction}

Colporrhexis causing separation of cervix from the vaginal fornix is an unanticipated, but perilous complication of labor. It can be life-threatening and easily missed, with the diagnosis being overlooked. Majority of cases of primary colporrhexis are of traumatic origin. The etiology of the infrequent primary spontaneous colporrhexis as in our case is mostly hypothetical. While secondary colporrhexis is reported along with vaginal birth after caesarean section, a reported case of primary colporrhexis after previous caesarean section is apparently rare. We hereby present one such extremely rare case of primary, spontaneous, complete colporrhexis during precipitate vaginal birth after caesarean section.

\section{Case Report}

The patient was 32 year old, gravida 2, para 1 women who had a past history of cesarean delivery by low transverse incision, having delivered a
$3.8 \mathrm{~kg}$ healthy male child. Patient had an uneventful antenatal course and had made an informed decision for a planned vaginal birth after cesarean section (VBAC). She was admitted to the hospital at 39 weeks of gestation in latent phase of labor with a singleton fetus in left occipito-anterior (LOA) position. She progressed spontaneously from $3 \mathrm{~cm}$ to full dilatation in one hour and within 15 minutes she had a spontaneous vaginal delivery with right medio-lateral episiotomy of a singleton live healthy female baby of $3.2 \mathrm{~kg}$ with Apgar 9 at 1 minute. The labor was not induced or augmented, however the patient mentioned eating date fruits.

Immediately after delivery of the baby and complete placenta, there was excessive vaginal bleeding. As per Advanced Life Support in Obstetrics (ALSO) guidelines [1], a second large bore intravenous line, continuous monitoring of vital signs, bimanual uterine massage, oxytocin infusion, intramuscular methylergonivine, misoprostol 1 mgm per rectal, blood group typing 
and cross match etc. were implemented. Her vitals were stable and uterus was contracted, but still she was having profuse vaginal bleeding. Exploration of the lower genital tract revealed a long midline laceration over the posterior vaginal wall extending to the posterior fornix with suspicion of avulsion of vaginal vault posteriorly. There was no extension of the episiotomy. Decision was made for exploration under anesthesia, as she was not under epidural.

Patient was transferred to the operation theatre. The genital tract was thoroughly and systematically explored under general anesthesia and confirmed the previous finding of the midline posterior vaginal wall tear. The previous uterine scar and cervix were intact. The rectum and anal canal were unaffected. Attempt was made to suture this rent vaginally, but its apex was opening into the pouch of Douglas (POD) and was not accessible. A decision for laparotomy was made. Intra-operatively, there was hemoperitoneum. The previous uterine scar and bladder were undamaged. There was midline tear seen in the inferior aspect of POD extending down to the previously noted vaginal wall tear. Posterior lip of the cervix was intact but exposed through the avulsed posterior vaginal vault. Both uterine vessels and ureters were intact. Edges of the tear were grasped by Allis forceps and anatomy restored by first layer of continuous and second layer of interrupted sutures. Vagina was re-explored. The total estimated blood loss was approximately $2000 \mathrm{ml}$. Patient was shifted to intensive care unit (ICU) for close monitoring. Patient received five units of packed red blood cells and four units of fresh frozen plasma. After 24 hours she was shifted to the ward and monitored closely when she developed mild right lung atelectasis and pleural effusion which was managed conservatively. She was discharged after four days.

On her review one week later in the outpatient department (OPD), she was stable, wound was healing well and there was slight stricture at the level of mid vagina. On her follow- up two months later in OPD, she reported normal sexual relationship without any dyspareunia or mechanical difficulty during sexual act. The patient was recommended and counseled for elective cesarean section in the future pregnancy.

\section{Discussion}

Obstetric hemorrhage emerges as the most common cause of severe maternal morbidity in almost all maternal 'near misses' and mortality audits in both developed and developing countries [2]. Primary postpartum hemorrhage $(\mathrm{PPH})$ occurring within 24 hours of delivery is the leading type of major obstetric hemorrhage [3]. The most common etiology of primary PPH is uterine atony, followed by genital tract trauma. Predisposing factors to trauma include parity, operative delivery, birth weight and precipitate labor; meanwhile episiotomy does not protect against severe laceration's occurrence [4].

Vaginal birth after cesarean section is evidence based good practice in obstetrics, it could be associated with a low percentage of rupture uterus or scar dehiscence, yet to our knowledge it is not associated with primary colporrhexis. The antenatal and intrapartum care of this patient complied with that recommended by the green-top guideline for vaginal birth after cesarean section [5]. Precipitate labor is an unusually short labor, which culminates in delivery of the fetus within three hours of onset of active labor [6]. Precipitate birth can lead to extensive lacerations of the cervix, vagina, vulva, or perineum or uterine rupture, postpartum atonic uterus, increased need for blood transfusion and longer duration of hospital stay [7]. In our case, it resulted in primary, spontaneous, complete colporrhexis.

Colporrhexis [8,9], otherwise referred to as rupture of the vaginal vault, causing separation of cervix from the vaginal fornix is a rare, but dangerous complication of labor. Primary colporrhexis is defined as a tear limited to the vaginal vault without cervical or uterine extension. 
This type constitutes merely $2.5 \%$ of the entire uterine and vaginal ruptures, whilst $97 \%$ of vaginal vault ruptures have concomitant extension into the cervix or lower uterine segment and are catalogued under secondary colporrhexis. Excessive retraction of the uterus can tear the cervix from its vaginal attachment resulting in spontaneous colporrhexis [10]. Those of traumatic origin often result from unskillful obstetric maneuvers [8-10]. Complete colporrhexis involves the entire vaginal wall thickness including the overlying peritoneum; while in incomplete variety, only the vaginal mucosa and muscularis are involved $[8,9]$.

In our case the rapid progress and very short second stage of labor led to shearing effect of the descending head on the upper posterior vaginal wall and did not affect the external or the internal anal sphincters. Whether, the previous pregnancy where there was persistent occipito-posterior position of fetal head at full dilatation without progress caused a weakening of vaginal vault is a matter of speculation. Colporrhexis is a surgical emergency. Inspite of the fact that such tears may be unsuspected, they usually present as hematomas or brisk hemorrhage as in our case. Meticulous exploration of such extensive vaginal vault tears is warranted. If tear is accessible, repair from below will suffice. In case of any suspicion of peritoneal perforation or of retroperitoneal or intra-peritoneal hemorrhage, there should be no hesitancy in considering laparotomy along with resuscitative measures. With such extensive damage, intrauterine exploration for possible rupture, especially as in our case of VBAC, is also indicated. Surgical repair along with simultaneous zealous blood replacement, effectual analgesia or anesthesia and adept assistance are imperative [11].

Injudicious and aggressive use of pharmaceutical uterine muscle stimulators as well as the widespread use of traditional preparations of pressed date fruits, castor oil and other traditional drinks in different parts of the world, can provoke strong uterine contractions that lead to precipitate labor [12], which can subsequently predispose to severe maternal morbidity. Date fruits and other traditional beverages are commonly used in Gulf areas to stimulate and initiate labor pains [12]. Few studies done to cover this area reported that date fruits facilitate and increase rate of cervical dilatation. However, more studies are needed before any conclusive evidence can be drawn.

"Maternal Near Miss" is defined as severe obstetrical complication that nearly caused maternal death, but the woman survives either due to a stroke of luck or due to appropriate and timely medical care [13]. There are many classifications of near miss based on organ failure, admission to ICU and on the type of complication. Our case fulfills the criteria of near miss as regards to blood loss, need for blood transfusion and admission to ICU. Near miss reporting and analyzing has been established as an appropriate tool for evaluation and measurement of quality of care provided in obstetrics. The 'three delays model' was developed for more comprehensive analysis of causes and preventability strategies to avoid or to minimize the long run complications of near miss. This model is composed of three phases: phase I - delay in decision to seek care by the individual and/or family, phase II - delay in reaching an adequate health care facility, and phase III - delay in receiving adequate care at the health facility. Phase III delay in terms of early diagnosis of the complications and time to start appropriate management are crucial factors in minimizing the mortality and morbidity [14].

In our case the systematic approach of diagnosis of the complications following the guidelines, allowed early diagnosis of the colporrhexis within few minutes following delivery, facilitating an early recourse to examination under anesthesia. As it was impossible to approach the fornix vaginally, an early decision was made for laparotomy. This double approach aided in achieving complete hemostasis and to restore anatomy. It took about three hours to control bleeding and to stabilize the patient. It needs to 
be emphasized that the pattern of management of the case, dependent on the woman being cared for in a consultant-led maternity unit with early and proper diagnosis of near miss, access to laboratory and blood bank facilities, skilled obstetric and anesthetic staff readily available in-house, well equipped operation theatre and availability of essential medicines are all key stones in saving the patient's life [15].

\section{Conclusion}

A case of primary, spontaneous, complete, intrapartum colporrhexis caused by precipitate vaginal birth after previous caesarean section is presented. The rareness of this unforeseen condition is emphasized. Also, to be stressed is the fact that such rare cases of maternal "near-misses" need well trained obstetrical and anesthetic team and fully equipped theatre to prevent maternal death and to minimize maternal morbidity.

Acknowledgment: Nursing staff in labor room and operation theatre along with anesthetist Dr. Mohammed Alhashmi for their support.

Contributors: The suggestion of publishing this case study arose in a group discussion among all the authors. The first version of the manuscript was drafted by Dr. Asmaa Abdulsalam and then complemented with suggestions made by all the other authors. All authors constituted the medical and surgical team that took care of the case.

Funding: None; Competing interests: None stated.

\section{References}

1. Anderson J, Etches D. Postpartum hemorrhage: third stage pregnancy. In: Advanced Life Support in Obstetrics (ALSO) Provider manual. 2012 ed. Chapter J, pp. 1-9.

2. Bashour H, Saad-Haddad G, DeJong J, Ramadan MC, Hassan S, Breebaart M, et al. A cross sectional study of maternal 'near-miss' cases in major public hospitals in Egypt, Lebanon, Palestine and Syria. BMC Pregnancy Childbirth. 2015;15:296.

3. Arulkumaran S, Mavrides E, Penney GC, Aberdeen. Prevention and management of post-partum haemorrhage. RCOG Green-Top Guideline 52. May 2009. Available from: http://www.rcog.org.uk/womenshealth/clinical-guidance/prevention-and-management- postpartum-haemorrhage-green-top-52. Accessed on June 26, 2016.

4. Monteiro MV, Pereira GM, Aguiar RA, Azevedo RL, Correia-Junior MD, Reis ZS. Risk factors for severe obstetric perineal lacerations.International Urogynecology Journal. 2016;27(1):61-67.

5. Gupta JK, Smith GCS, Chodankar RR. Birth after previous caesarean birth. Royal College of Obstetricians and Gynaecologists Green-top Guideline. October 2015;45.

6. Suzuki S. Clinical significance of precipitous labor. Journal of Clinical Medicine Research. 2015;7(3):150153.

7. Sheiner E, Levy A, Mazor M. Precipitate labor: higher rates of maternal complications. European Journal of Obstetrics, Gynecology, and Reproductive Biology. 2004;116(1):43-47.

8. Sharma KKY, Frith KM. Primary complete colporrhexis in a primigravida. British Journal of Obstetrics and Gynaecology. 1993;100(12):1142-1144.

9. Wijesingbe PS. Primary spontaneous complete colporrhexis: The Ceylon Medical Journal. 2002;47(1):29.

10. Sakhare AP, Bhanap PL, Mahale AR. Bowel prolapse through colporrhexis - a complication of home delivery. The Journal of Obstetrics and Gynecology of India. 2007;57(6):553-554.

11. Pritchard T. Obstetrical hemorrhage. In: Cunningham FG, Leveno KJ, Bloom SL, Spong CY, Dashe JS, Hoffman BL et al. William's Obstetrics. 24 $4^{\text {th }}$ ed. New York: Mc Graw-Hill; 2014. p.789.

12. Al-Kuran O, Al-Mehaisen L, Bawadi H, Beitawi S, Amarin Z. The effect of late pregnancy consumption of date fruit on labour and delivery. Journal of Obstetrics and Gynaecology. 2011;31(1):29-31.

13. Filippi V, Ronsmans C, Gohou V, Goufodji S, Lardi M, Sahel A, et al. Maternity wards or emergency obstetric rooms? Incidence of near-miss events in African hospitals. Acta obstetrician etgynecologica Scandinavica. 2005;84(1):11-16.

14. Pacheco AJ, Katz L, Souza ASR, de Amorim MM. Factors associated with severe maternal morbidity and near miss in the São Francisco Valley, Brazil: a retrospective, cohort study. BMC Pregnancy and Childbirth. BMC series. 2014;14:91.

15. Pacagnella RC, Cecatti JG, Parpinelli MA, Sousa MH, Haddad SM, Costa ML, et al. Delays in receiving obstetric care and poor maternal outcomes: results from a national multicentre cross-sectional study. BMC Pregnancy and Childbirth. BMC Series. 2014;14:159. 